\title{
Les objectifs des « réseaux futurs » VUS PAR L'UIT
}

\author{
Jean-Michel MUR \\ Président d'honneur du Club \\ fibres optiques et réseaux \\ jm.mur@orange.fr
}

Pour faire face à l'explosion de la demande en débits et la diversité des services souhaités, I'Union internationale des télécommunications a développé le concept de «Future Networks» (réseaux futurs) visant à remplacer les actuels «réseaux de prochaine génération» (Next Generation Networks). Panorama des principaux objectifs fixés à ces futurs réseaux de communication...

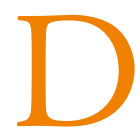
epuis plus d'un siècle, la branche Télécommunications de l'Union internationale des télécommunications (UIT-T) a permis et permet, à travers ses travaux et ses recommandations, le déploiement et l'interconnexion de l'ensemble des réseaux de communication mondiaux dont ceux en fibres optiques. De grandes étapes sont caractéristiques des évolutions de ces réseaux (cf.enca$d r e ̂)$. Voici quelques exemples depuis les années 1990, où l'UIT-T a prôné de grands choix technologiques et architecturaux pour le déploiement des réseaux. À partir de 1998, ce fut le concept «Infrastructure mondiale de l'information » (Global information infrastructure). Puis, au début des années 2000, le protocole Internet s'est déployé à travers les recommandations de l'UIT-T traitant des « Aspects du protocole Internet » (Internet protocol aspects). À partir de 2004, c'est l'arrivée des réseaux appelés « Réseaux de prochaine génération » (Next Generation Networks-NGN). Et, quelques années après, dès 2011, l'UIT-T a débuté les premières réflexions sur les réseaux du futur qu'elle a appelés Future Networks $(\mathrm{FN})$, soit réseaux futurs (RF), ainsi que sur l'informatique en nuage (Clond computing). Et, vers la mi-2016, c'est la publication des recommandations sur l'Internet des objets, villes et communautés intelligentes (Internet of things and smart cities and communities) et la publication de la feuille de route Big data standardization roadmap.

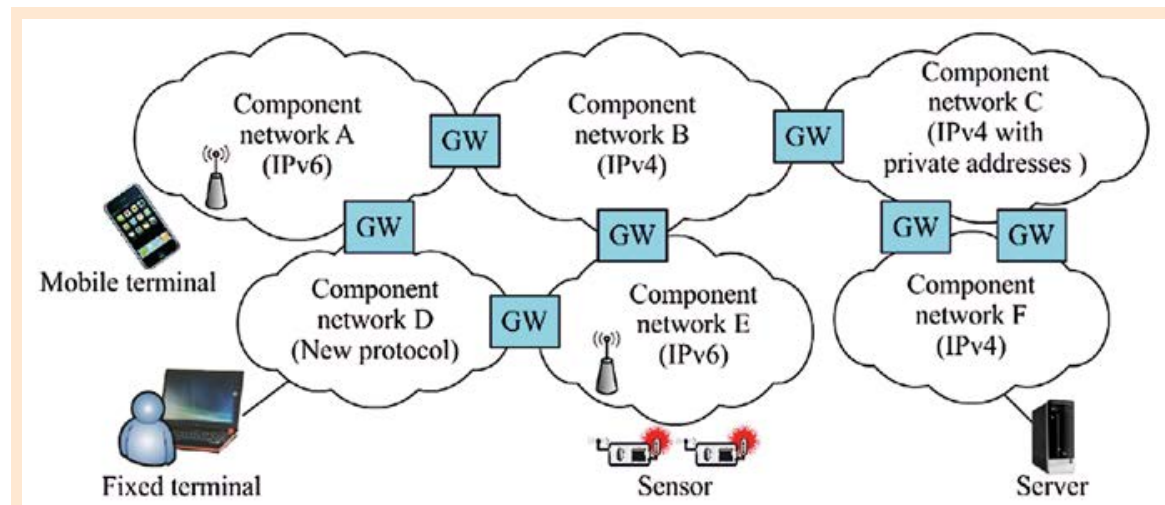

Figure 1. Environnement d'un réseau avec des composants réseaux hétérogènes (GW : gateway). Crédit: UIT-T, Y.3034, p. 12

Pour l'UIT-T, la décennie 2015-2025 est ainsi celle de ces réseaux futurs ${ }^{1}$. Mais, que sont ces réseaux futurs vus par l'UIT-T ? Quels sont les objectifs que l'UIT-T leur a fixés? Quelques éléments de réponse...

\section{Que sont \\ les «réseaux futurs »?}

La recommandation générale, Y.3001, publiée en français en mai 2011 et intitulée «Réseaux futurs : Objectifs et but de conception " précise que «...il est supposéque la date de mise en place des réseaux futurs est fixée entre 2015 et 2020 environ » et qu'un réseau futur (RF) pourra fournir des services, capacités et fonctionnalités qu'il serait difficile d'offrir par les technologies de réseau existantes. Le choix du pluriel
« réseaux futurs » tient au fait qu'il peut y avoir plusieurs définitions de l'expression « réseau futur ». L'UIT-T précise : «Un réseau futur pent-être: d'un type a) tel un réseau-élément nouveau ou une version améliorée d'un réseau-élément existant, ou d'un type b) comme un ensemble hétérogène de réseaux-éléments nouveaux ou de réseaux-éléments nouveaux et existants, ensemble exploitéen tant que réseau unique. » (cf. figure 1).

Par réseau-élément, l'UIT-T entend un réseau homogène unique mais qui ne peut pas constituer à lui seul une infrastructure de télécommunication complète de bout en bout. Et, un réseau de type b) peut également comporter des réseaux de type a). De plus, il est à noter que, dans le contexte de cette recommandation Y.3001, un réseau-élément est qualifié de 
« nouveau » lorsqu'il permet la fourniture des services, capacités et fonctionnalités difficiles ou impossibles à fournir au moyen des technologies de réseau existantes. Enfin, ces RF devraient pouvoir assurer l'universalisation des services, c'est-à-dire la mise en place de processus permettant la fourniture des services de télécommunication à toutes les personnes ou à tous les groupes de personnes indépendamment de leur situation sociale, géographique et économique.

\section{Quels sont les objectifs des RF?}

Afin que ces réseaux futurs se distinguent des réseaux existants, l'UIT-T a défini quatre objectifs généraux et douze objectifs relatifs à leur conception (cf. figure 2). Les quatre objectifs généraux sont liés aux services, aux données, à l'environnement et à la dimension socio-économique. Pour la prise en charge des services, dont le nombre, la variété et les fonctionnalités croissent, l'UIT-T recommande que les RF prennent en charge leur fourniture tout en limitant les hausses des coûts de déploiement et d'exploitation. Pour l'accès aux données, que ce soit des contenus audio, vidéo ou autres, l'architecture des RF devra être optimisée pour faire face à l'explosion de la demande tout en assurant sécurité et rapidité d'accès. En ce qui concerne les considérations environnementales, la conception et l'installation des RF devront limiter la consommation de matière et d'énergie tout en réduisant l'émission des gaz à effet de serre. Enfin, les RF devront prendre en compte les questions socio-économiques et les différents acteurs jouant dans l'écosystème des réseaux tout en réduisant les coûts d'utilisation, en favorisant déploiement et durabilité et en aidant à une large distribution des services.

Les quatre objectifs généraux fixés aux RF s'appuient sur douze objectifs relatifs à leur conception : diversité des services, souplesse fonctionnelle, virtualisation des ressources, accès aux données, consommation d'énergie, universalisation des services, incitations économiques, gestion des réseaux, mobilité, optimisation, identification, fiabilité et sécurité. Ces objectifs liés à la conception des RF concernent des capacités et caractéristiques de haut niveau tout en sachant que certaines d'entre elles pourraient être difficilement prises en charge dans un RF donné, voire ne serait pas implémentées. Quelques explications de l'UIT-T sur ces objectifs relatifs à la conception des RF...

\section{Objectifs relatifs à la conception}

Concernant la diversité des services, les RF devront prendre en charges de nouveaux services et applications différents dans leurs caractéristiques - latence, largeur de bande, de quelques octets occasionnellement à plusieurs Gbit/s voire au Tbit/s en continu... ; dans leur comportement de trafic sécurité, fiabilité et mobilité ; dans la variété des dispositifs de communication - capteurs, télédétecteurs, terminaux fixes, mobiles, vidéo à très haute résolution... ; dans le canal de transmission, qu'il soit dédié ou virtuel, etc.

Pour la souplesse fonctionnelle, les RF devront favoriser le déploiement des nouveaux services demandés par les utilisateurs et assurer leur maintenance. En effet, il est difficile de prévoir les futures demandes à long terme. Ainsi, les RF devront faire preuve de polyvalence, accepter des modifications dynamiques des fonctionnalités, de nouveaux protocoles de transmission ou des services expérimentaux puis généralisés.

Dans le domaine des réseaux, il faut distinguer les réseaux physiques et leur virtualisation. Ainsi, les RF devront permettre la virtualisation des ressources qui leur sont associées afin qu'elles puissent être subdivisées. Les grands types de ressources physiques concernent le transport avec des nœuds de réseaux, liens, routeurs, commutateurs..., mais aussi les ressources de calcul, les serveurs, les unités de stockage de données, etc. Avec la virtualisation, une ressource unique

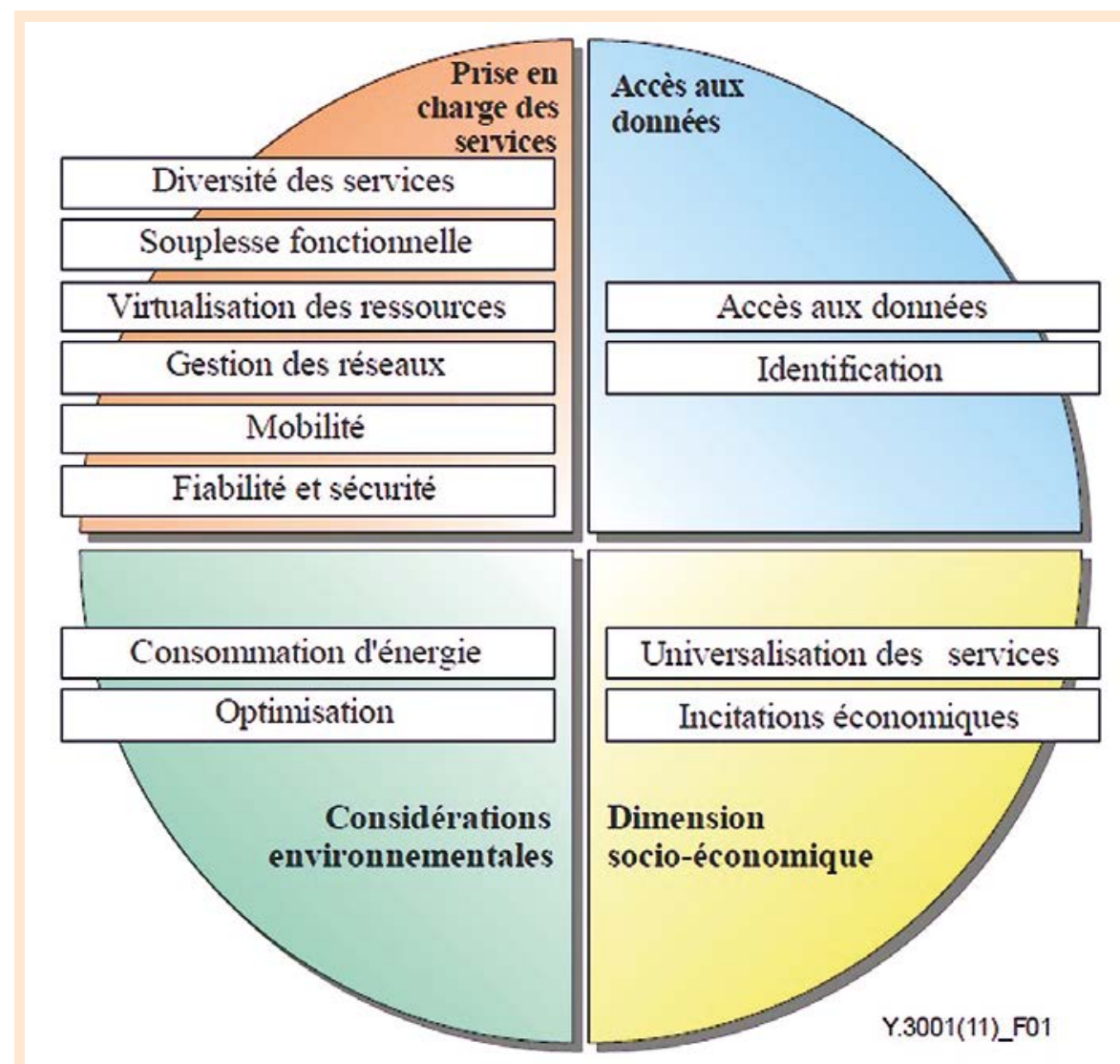

Figure 2. Les réseaux futurs : quatre objectifs généraux et douze objectifs de conception. Crédit : UIT-T, Y.3001, p. 11 


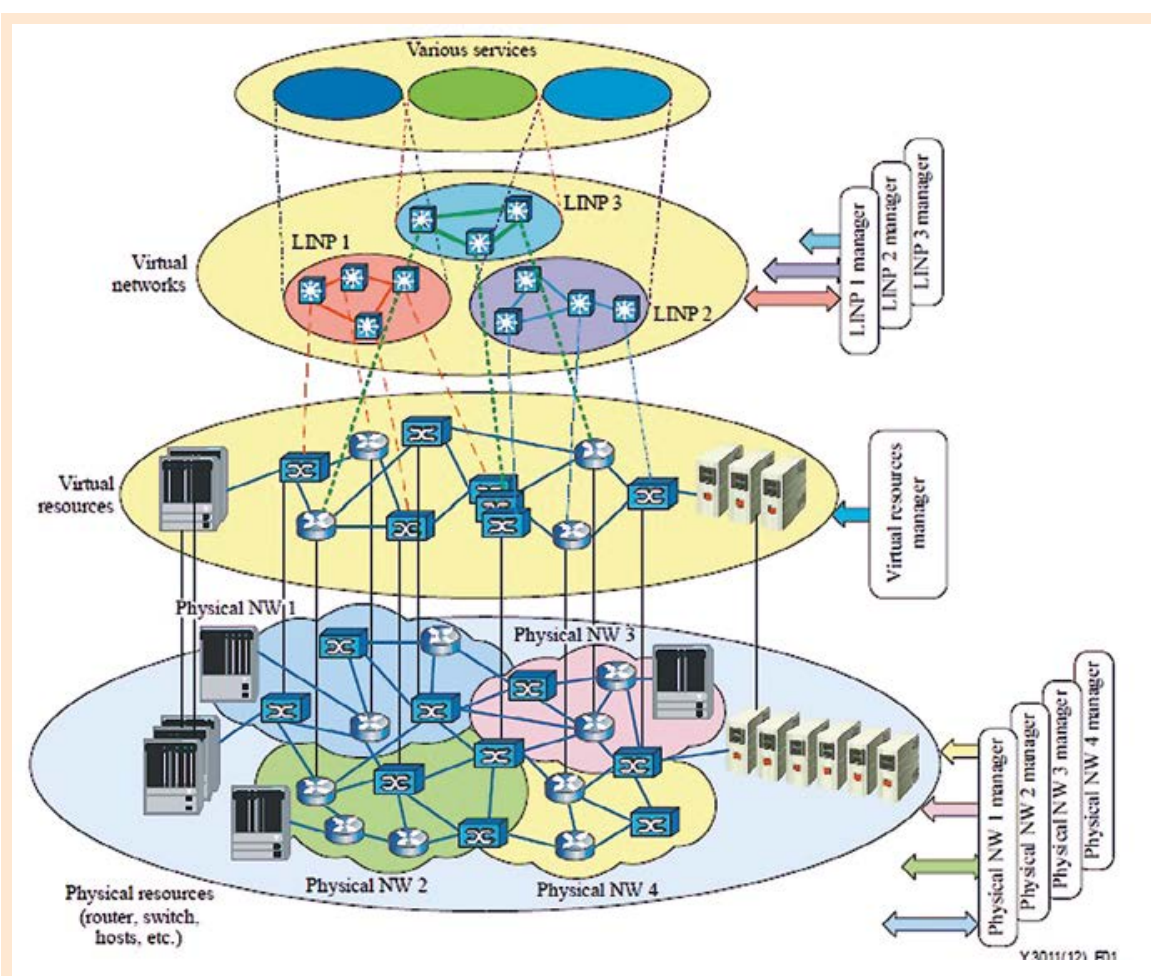

Figure 3. Concept architectural de la virtualisation de réseaux. LINP : logically isolated network partition, subdivision de réseau logiquement isolée. Crédit : UIT-T, Y.3011, p. 9

pourra être partagée simultanément par plusieurs ressources dites virtuelles comme un réseau privé virtuel (virtual private network - VPN), un réseau local virtuel (virtual local area network VLAN), etc. En quelque sorte, c'est le concept d'une subdivision de réseau logiquement isolée (logically isolated network partition - LINP), c'est-à-dire un réseau de ressources virtuelles dynamiquement reconfigurables qui, pour l'utilisateur, est vu comme un réseau sans virtualisation (cf. figure 3).

Le cas de l'accès aux données, offre une double problématique pour les RF : traiter de façon optimale et efficace des volumes considérables de données et être dotés de mécanismes d'extraction des données rapidement, quel que soit leur emplacement. En effet, les utilisateurs recherchent des données via des mots-clés et y accèdent sans connaître leur emplacement physique. Pour cela, on trouve des variantes comme les réseaux de distribution de contenus (content distribution network - CDN) ou des réseaux pair-à-pair (peer-to-peer - P2P). Ainsi, les réseaux sont utilisés comme outils d'accès aux données. Ce rôle est à conserver par les RF mais, en raison de l'informatique dématérialisée, il faut concevoir une future méthode d'acheminement qui reposerait sur le nom des données ou des contenus stockés dans des emplacements multiples, le tout servi par un mécanisme de mise en mémoire tampon sur l'ensemble du réseau. À ne pas négliger, la sécurité qui pourrait être renforcée en donnant une signature de clé publique aux données et contenus, ce qui prouverait leur authenticité.

Quant à l'objectif d'économie d'énergie, la conception des RF devra faire preuve d'une conscience environnementale en veillant à l'utilisation de techniques économes en énergie que ce soit lors de l'installation du RF ou durant son exploitation. À ce titre, de nombreux équipements de réseau, à tous les niveaux, sont concernés : routeurs, commutateurs, multiplexeurs, unités de réseau optique (optical network unit - ONU), cartes d'interface avec le réseau, passerelles domestiques (box), etc. Parmi les pistes à suivre, on trouve l'intégration des composants (large scale integration - LSI), les débits de liaison adaptatifs (adaptive link rate - ALR), les réglages dynamiques de tension (dynamic voltage scaling - DVS), etc. En contrepartie de leurs empreintes environnementales, les RF sont utiles pour la diminution de l'impact d'autres domaines sur l'environnement. Deux exemples : la mise en place d'un réseau électrique dit intelligent pour l'optimisation de la distribution d'électricité,

ou bien le déploiement de réseaux de

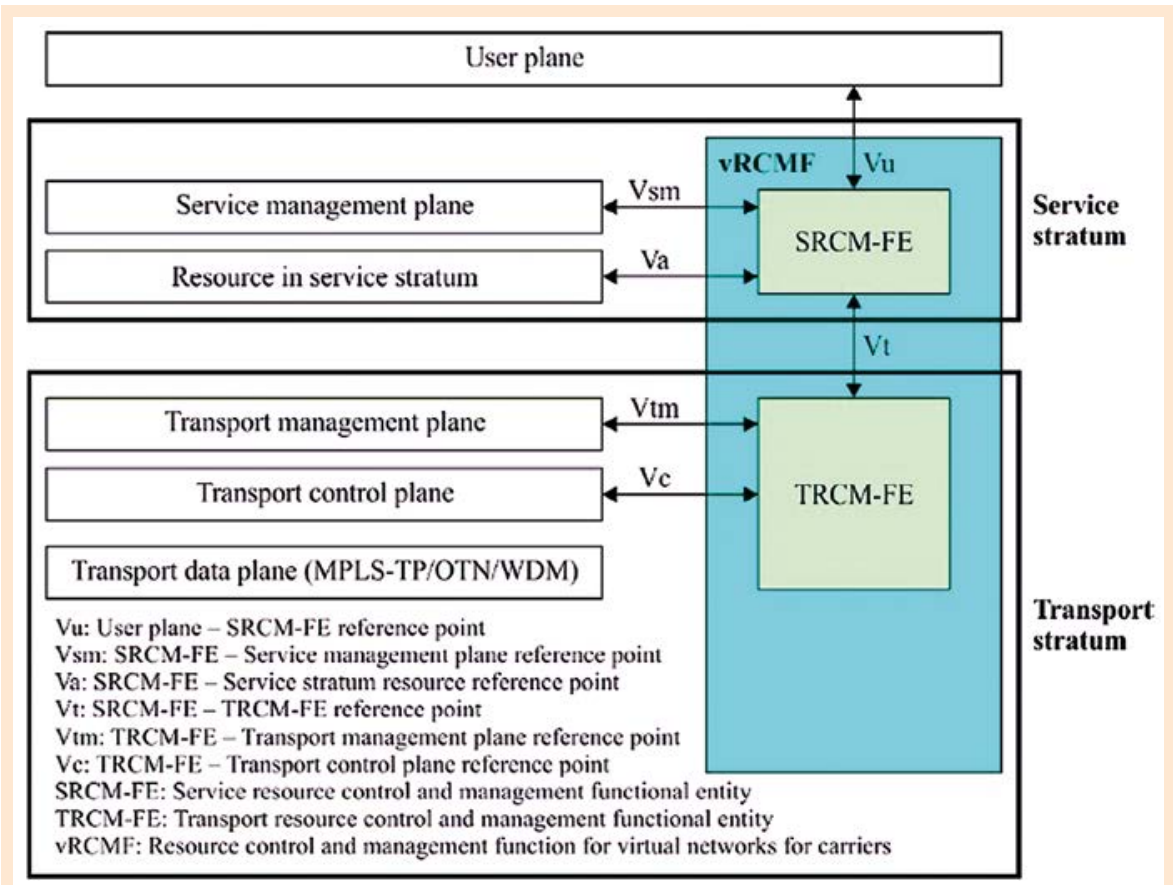

Figure 4. Architecture de gestion et contrôle des ressources de réseau virtuel pour opérateurs. Crédit : UIT-T, Y.3014, p. 10 


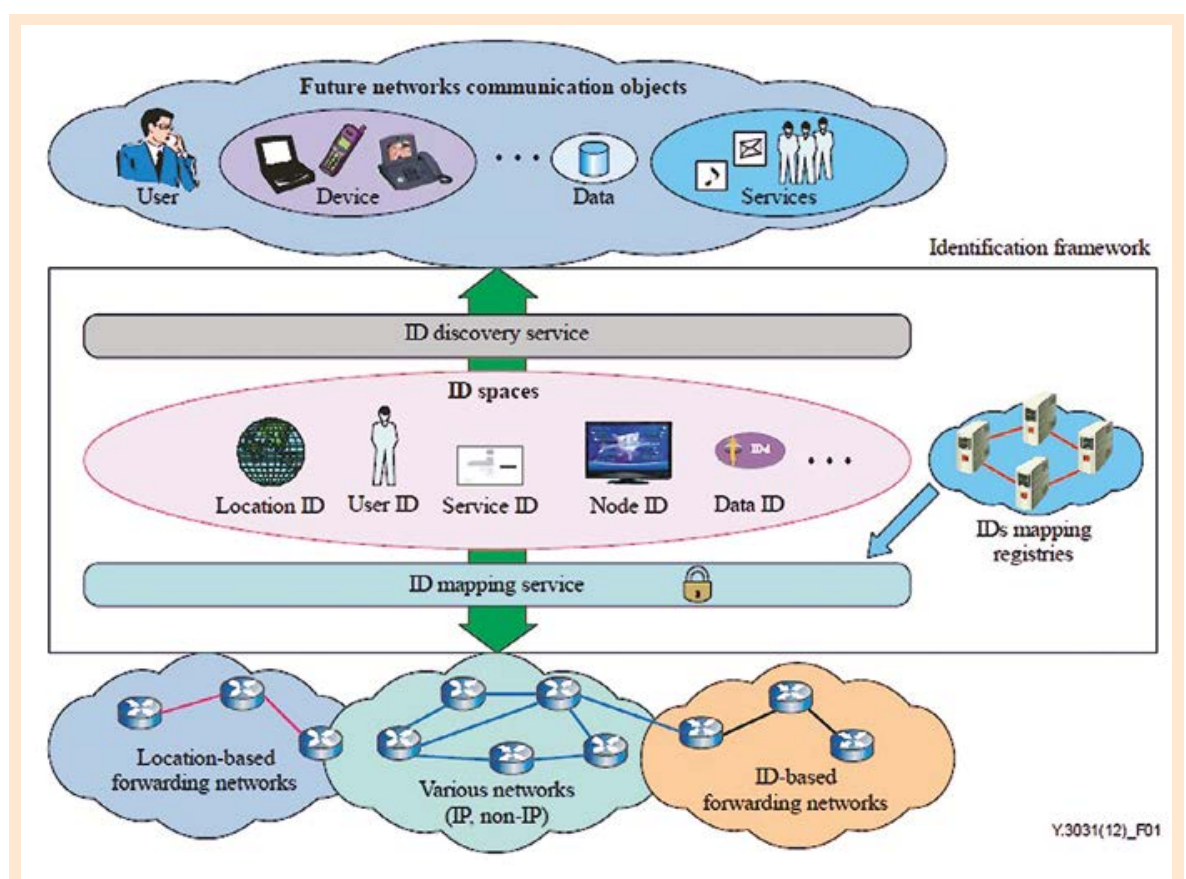

Figure 5. Cadre d'identification dans les RF. Crédit : UIT-T, Y.3031, p. 12 répondre aux besoins de transport des VNC. La couche de transport est, bien évidemment, multicouches comme le transport du protocole Internet (IP) sur un multiplexage par répartition en longueurs d'onde dense (dense wavelength division multiplexing-DWDM), ou IP sur le mode de transport multi-protocolaire par étiquetage (multiprotocollabel switching-transportprofile-MPLS-TP), ou IP sur un réseau de transport optique (optical transport network - OTN)... tout un monde de combinaisons possibles.

Un dernier mot pour ne pas oublier les autres objectifs liés à la conception des RF que sont l'universalisation des services, les incitations économiques, la mobilité, l'optimisation, l'identification (cf.figure 5), la fiabilité et la sécurité. De belles perspectives de recherche et développement pour la décennie 2015-2025 grâce aux RF. capteurs ubiquitaires (ubiquitous sensor network - USN) pour la surveillance de températures ou autres; en quelque sorte, c'est le « passage au vert » grâce aux RF.

Sans être exhaustif sur les douze objectifs de conception des RF, terminons par la gestion des réseaux. En effet, c'est un problème rencontré dans les réseaux actuels qui sont confrontés au fait que les systèmes d'exploitation et de gestion ont été conçus spécifiquement pour chaque élément de réseau. Ainsi, la multiplication des fonctionnalités va accroître la complexité et faire augmenter les coûts d'exploitation. D'où l'objectif, pour les RF, de fournir des systèmes d'exploitation et de gestion efficaces par l'intermédiaire d'interfaces de gestion plus intégrées. À titre d'exemple, on peut s'intéresser au concept de réseau virtuel d'opérateur (virtual network for carrier - VNC). Pour ce cas, la figure 4 présente une architecture de gestion et de contrôle. Les deux entités fonctionnelles, celle de gestion et de contrôle des ressources de services (service resource control and management-functional entity - SRCM-FE) et celle de gestion et de contrôle du transport (transport resource control and management-functional entity - TRCM-FE) interagissent l'une avec l'autre afin de

\section{Les grands choix prônés par l'UIT-T}

Les grands choix technologiques et architecturaux des réseaux prônés par l'UIT-T sont détaillés dans les recommandations de la série $Y$ qui couvre les grands domaines suivants.

Y.100-Y.999 : Global information infrastructure, infrastructure mondiale de l'information, dont la première recommandation date de 1998. Un des premiers buts poursuivis était l'interopérabilité des services et applications d'information. Y.1000-Y.1999 : Internet protocol aspects, aspects relatifs au protocole Internet, cadre proposé au début de l'année 2000 . Son objectif principal était la fourniture de services transparents à l'utilisateur dans le contexte de convergence entre réseaux IP et réseaux de télécommunications.

Y.2000-Y.2999 : Next Generation Networks, réseaux de prochaine génération, série de recommandations publiée à partir de 2004. L'objectif principal des NGN est de tenir compte de la déréglementation des marchés, de l'explosion du trafic numérique et des services vidéo, couplés à une mobilité générale, à la convergence des réseaux et à la confluence des services.

Y.3000-Y.3499 : Future Networks (FN), réseaux futurs (RF), au déploiement prévu entre 2015 et 2020 avec quatre objectifs liés aux services, aux données, à l'environnement et à la dimension socio-économique. Parmi ces RF, il faut noter les réseaux ubiquitaires intelligents (Smart Ubiquitous Network - SUN) et les réseaux de capteurs ubiquitaires (Ubiquitous Sensor Network - USN).

Y.3500-Y.3999 : Cloud computing, informatique en nuage, dont les premières recommandations ont été publiées en 2014 , en relation avec les normes de la CEl et de l'OSI.

Y.4000-Y.4999 : Internet of things and smart cities and communities, Internet des objets, villes et communautés intelligentes. Peu de recommandations de l'UIT-T sur ce sujet, mais plusieurs « Suppléments » - Y.Sup27 à Y.Sup39 - sur les villes et Y.Sup40 « Big data standardization roadmap » de juillet 2016.

Toutes ces recommandations, dont certaines en français, sont téléchargeables - gratuitement pour celles ayant plus de six mois, en payant pour les autres à l'adresse : www.itu.int/rec/T-REC-Y 